\title{
Optimizing Workflows for Fast and Reliable Metabolic Tumor Volume Measurements in Diffuse Large B Cell Lymphoma
}

Coreline N. Burggraaff $\odot,{ }^{1}$ Fareen Rahman, ${ }^{2}$ Isabelle Kaßner, ${ }^{3}$ Simone Pieplenbosch, ${ }^{1}$ Sally F. Barrington, ${ }^{4}$ Yvonne W.S. Jauw, ${ }^{1,5}$ Gerben J.C. Zwezerijnen, ${ }^{5}$ Stefan Müller, ${ }^{3}$ Otto S. Hoekstra, ${ }^{5}$ Josée M. Zijlstra, ${ }^{1}$ Henrica C.W. De Vet, ${ }^{6}$ Ronald Boellaard, ${ }^{5}$ On behalf of the PETRA Consortium

\footnotetext{
${ }^{1}$ Department of Hematology, Cancer Center Amsterdam, Amsterdam UMC, Vrije Universiteit Amsterdam, De Boelelaan 1117, 1081HV, Amsterdam, Netherlands

${ }^{2}$ Department of Clinical Oncology, Guy's and St Thomas' NHS Foundation Trust, Guy's Cancer, London Bridge, London, UK

${ }^{3}$ Department of Nuclear Medicine, University Hospital Essen, University of Duisburg-Essen, Forsthausweg 2, 47057, Duisburg, Germany

${ }^{4}$ King's College London and Guy's and St Thomas' PET Centre, School of Biomedical Engineering and Imaging Sciences, King's Health Partners, King's College London, London, UK

${ }^{5}$ Department of Radiology and Nuclear Medicine, Cancer Center Amsterdam, Amsterdam UMC, Vrije Universiteit Amsterdam, De Boelelaan 1117, 1081HV, Amsterdam, Netherlands

${ }^{6}$ Department of Epidemiology and Biostatistics, Amsterdam Public Health Research Institute, Amsterdam UMC, Vrije Universiteit Amsterdam, De Boelelaan 1105, 1081HV, Amsterdam, Netherlands
}

\begin{abstract}
Purpose: This pilot study aimed to determine interobserver reliability and ease of use of three workflows for measuring metabolic tumor volume (MTV) and total lesion glycolysis (TLG) in diffuse large B cell lymphoma (DLBCL).

Procedures: Twelve baseline $\left[{ }^{18} \mathrm{~F}\right] \mathrm{FDG}$ PET/CT scans from DLBCL patients with wide variation in number and size of involved organs and lymph nodes were selected from the international PETRA consortium database. Three observers analyzed scans using three workflows. Workflow A: user-defined selection of individual lesions followed by four automated segmentations (41\%SUVmax, A50\%SUVpeak, SUV $\geq 2.5, S U V \geq 4.0$ ). For each lesion, observers indicated their "preferred segmentation." Individually selected lesions were summed to yield total MTV and TLG. Workflow B: fully automated preselection of $\left[{ }^{18} \mathrm{~F}\right] \mathrm{FDG}$-avid structures (SUV $\geq 4.0$ and volume $\geq 3 \mathrm{ml}$ ), followed by removing non-tumor regions with single mouse clicks. Workflow $\mathrm{C}$ : preselected volumes based on Workflow B modified by manually adding lesions or removing physiological uptake, subsequently checked by experienced nuclear medicine physicians. Workflow $\mathrm{C}$ was performed 3 months later to avoid recall bias from the initial Workflow B analysis. Interobserver reliability was expressed as intraclass correlation coefficients (ICC).
\end{abstract}

Electronic supplementary material The online version of this article (https:// doi.org/10.1007/s11307-020-01474-z) contains supplementary material, which is available to authorized users. 
Results: Highest interobserver reliability in Workflow A was found for $S U V \geq 2.5$ and $S U V \geq 4.0$ methods (ICCs for MTV 0.96 and 0.94 , respectively). SUV $\geq 4.0$ and A50\%Peak were most and SUV $\geq 2.5$ was the least preferred segmentation method. Workflow B had an excellent interobserver reliability $(I C C=1.00)$ for MTV and TLG. Workflow $C$ reduced the ICC for MTV and TLG to 0.92 and 0.97 , respectively. Mean workflow analysis time per scan was 29,7 , and 22 min for A, B, and C, respectively.

Conclusions: Improved interobserver reliability and ease of use occurred using fully automated preselection (using SUV $\geq 4.0$ and volume $\geq 3 \mathrm{ml}$, Workflow B) compared with individual lesion selection by observers (Workflow A). Subsequent manual modification was necessary for some patients but reduced interobserver reliability which may need to be balanced against potential improvement on prognostic accuracy.

Key Words: Diffuse large B cell lymphoma, Metabolic tumor volume, PET/CT, Total lesion glycolysis

\section{Introduction}

In young patients with diffuse large $\mathrm{B}$ cell lymphoma (DLBCL), a large maximum tumor diameter is an indicator of poor prognosis [1]. Recent progress in lymphoma care has recommended exploration of the prognostic value of volumetric tumor bulk measured on staging 2-deoxy-2$\left[{ }^{18} \mathrm{~F}\right]$ fluoro-D-glucose $\left(\left[{ }^{18} \mathrm{~F}\right] \mathrm{FDG}\right) \mathrm{PET} / \mathrm{CT}$, with methods combining metabolic activity and volume [2]. In lung cancer patients, studies have focused on finding the most reliable tumor segmentation method [3-5]. However, compared with lung cancer, lymphoma segmentation is more challenging due to higher number of lesions, multiple anatomical locations, and inter- and intratumoral $\left[{ }^{18} \mathrm{~F}\right] \mathrm{FDG}$ uptake heterogeneity.

Preliminary data suggest that baseline metabolic tumor volume (MTV) has a prognostic value in DLBCL [6-9] and predict outcome better than bulky disease measured by maximum tumor diameter [7]. Total lesion glycolysis (TLG) - defined as SUVmean in a volume multiplied by the corresponding MTV—seems to perform similarly [7] or inferiorly $[6,8]$ in predicting outcome of DLBCL patients. Various segmentation methods to measure MTV and TLG are being used in clinical lymphoma studies [10]: most use a fixed SUV threshold (e.g., $\mathrm{SUV} \geq 2.5[7,9]$ or $\mathrm{SUV} \geq 4.0$ [11]) or a percentage of SUVmax (e.g., $41 \%$ of SUVmax [6, 8, 12]) to define MTV. An important finding from earlier studies in DLBCL is that optimal cutoff values range widely (220-550 ml), probably because of using different methodologies, small patient cohorts, differences in patient risk factors, and therapies [13]. Moreover, these data-driven cutoff values should be interpreted with caution, as they depend highly on acquisition and reconstruction protocols.

Segmentation methods in these studies are generally derived from phantom experiments [4, 12], or correlation with pathological specimens in lung cancer [4]. Limited data are available about the differences in ease of use in the lymphoma clinical setting and interobserver reliability of these tumor segmentation methods [10]. Previous studies in
DLBCL [10], T cell [14], and Hodgkin lymphoma [15] showed that different segmentation methods, despite having different cutoff values, show comparable accuracy for predicting survival. Therefore, for future use in practice and clinical trials a robust, reliable and easy-i.e., with least required observer interaction-segmentation workflow is necessary. To the best of our knowledge, this is the first pilot study in DLBCL that compares interobserver reliability and ease of use of three workflows for measuring MTV and TLG, and that assesses the effect of manual modification on interobserver reliability.

\section{Materials and Methods}

\section{Study Population}

Twelve baseline $\left[{ }^{18} \mathrm{~F}\right] \mathrm{FDG}$ PET/CT scans from newly diagnosed DLBCL patients with wide variation in number and size of involved organs and lymph nodes lesions were selected from the international PETRA database (http:// www.petralymphoma.org). The use of all data within the PETRA imaging database has been approved by the Medical Ethics Review Committee of the VU University Medical Center (JR/20140414) after patients' consent to participate in the studies included in the database.

\section{Image Analysis Workflows $A$ and $B$}

Two semi-automated workflows (Workflows A and B) were performed in the same week, by three independent observers using the ACCURATE software tool [16]. Manual modifications of the semi-automatically generated volumes of interest (VOIs) were not allowed initially. The workflow with the best interobserver reliability and ease of use was selected as starting point for manual modification in Workflow $\mathrm{C}$.

Workflow A comprised a user-defined selection of individual lesions. The observers had to select individual lesions (by a single mouse click in the "hottest" part of each 
lesion), followed by automated segmentation in the tool using four separate frequently published segmentation methods:

1. $41 \%$ of SUVmax (41\%MAX)

2. A50\% of SUVpeak, i.e., $50 \%$ of SUVpeak with local background correction [17] (A50\% $\mathrm{P})$

3. fixed SUV threshold of 2.5 ( $\mathrm{SUV} \geq 2.5$ )

4. fixed SUV threshold of $4.0(\mathrm{SUV} \geq 4.0)$.

The four segmentation methods were initiated from one single click by the observer, to avoid introduction of extra variability by repeated clicking. Moreover, the tool first calculated a robust local maximum (using a region growing method applying a $70 \%$ threshold of the point clicked) in order to be less dependent on the exact point clicked by the observer. Generated VOIs were summed for all lesions selected by each observer to calculate MTV and TLG according to each of the four segmentation methods.

To explore the use and performance of consensus methods, two methods were added afterwards, which use the delineations found with the above four standard methods as input for a majority vote (MV) approach [18]. MV volumes were defined by all voxels included in the MTV or TLG by at least two (MV2) or three (MV3) of the input methods.

Workflow B consisted of a fully automated preselection of $\left[{ }^{18} \mathrm{~F}\right]$ FDG-avid structures defined by an $\mathrm{SUV} \geq 4.0$ and a volume threshold of $\geq 3 \mathrm{ml}$. These preselected regions resulted into an identical starting point for all observers but could include non-tumor regions with normal increased $\left[{ }^{18} \mathrm{~F}\right]$ FDG uptake, such as the brain or bladder. From this starting point, the observers decided on the removal of nontumor regions by using a clearing option (i.e., single click(s)) or spatial limits to reduce the analyzed field of view (e.g., using a slider option to exclude superior slices including the brain or inferior slices including the bladder); after this, only lymphoma lesions remain. Therefore, a region is defined as any preselected 3D-VOI with uptake above the SUV $\geq 4.0$ threshold, whereas a lesion is defined as a $3 \mathrm{D}-\mathrm{VOI}$ identified by the observer as lymphoma.

To determine ease of use for both workflows, each observer noted the total analysis time per patient (including loading of the scan, performing the analysis, and saving results).

In addition, the success of all semi-automatically generated VOIs was rated by each observer according to the following definitions:

- Failed: generated VOI is unrealistic or does not contain complete lesion

- Poor: generated VOI takes into account physiological uptake or contains a lot of background and manual modification is needed

- Acceptable: only minimal manual modification needed for good VOI
- Good: generated VOI is comparable to what you consider to be lymphoma

A mean "success rate" (all acceptable and good ratings) was calculated for each method. Finally, observers had to choose one "preferred segmentation" for the generated VOIs. The MV2 and MV3 consensus methods were rated by one experienced observer according to the same success definitions. As these MV methods were assessed afterwards, they could not be chosen as "preferred segmentation."

\section{Image Analysis Workflow C}

The observers used the fully automated method as in Workflow $\mathrm{B}$ for the analyses on the same twelve scans (Workflow C1). These analyses were performed 3 months later to minimize recall bias. In addition to the interactive deletion of physiological uptake regions similar to Workflow B, the observers were allowed in Workflow $\mathrm{C}$ to manually modify the generated VOIs by adding missed lesions (with the A50\%P option or manually) and removing of physiological uptake with an "eraser" tool. The manually modified MTVs and TLGs were checked for correct delineation and identification of tumor sites (and changed if needed) by independent nuclear medicine physicians (NM, one per observer) with more than 10 years of experience with $\left[{ }^{18} \mathrm{~F}\right] \mathrm{FDG}$ PET/CT evaluation in lymphoma (OSH, SFB, SM; Workflow C2).

\section{Statistical Analysis}

Success rates of generated VOIs were analyzed descriptively. Interobserver reliability was expressed as intraclass correlation coefficients (ICCs) and coefficients of variation $(\mathrm{CoVs})$. ICC estimates and their $95 \%$ confidence intervals $(95 \% \mathrm{CIs})$ were calculated with a two-way random-effects model for absolute agreement [19]. The $95 \%$ CIs of the ICC values were interpreted as poor $(<0.5)$, moderate $(0.5-0.75)$, good $(0.75-$ $0.9)$, and excellent $(>0.9)[20,21]$. CoV was calculated as the ratio of the standard deviation (over three observers) of MTVs or TLGs divided by the mean values per patient. Mean CoVs are presented, i.e., CoVs averaged over all patients. BlandAltman plots were drawn to visually assess potential bias of the mean differences between the workflows and to estimate $95 \%$ limits of agreement [22]. Normality of MTV and TLG differences before and after manual modification was checked with the Shapiro-Wilkinson (SW) test, in which $P<0.05$ was an indication of a non-normal distribution. Statistical analyses were performed using SPSS Statistics (IBM, v.20).

\section{Results}

\section{Workflow A; Individual Lesion Selection}

Lesion Selection The total number of selected lesions for observer 1,2, and 3 was 162,117 , and 118, respectively, which 
was due to the fact that observer 1 separately selected small lesions close to larger lesions, which were ignored by observers 2 and 3. It resulted in larger volumes for the $\mathrm{A} 50 \% \mathrm{P}$ and the 2 MV consensus methods for observer 1 (Supplemental Fig. 1). In total, 76 lesions were selected by all observers; of which, 35 showed identical segmentation results, and 18 lesions had a difference in volume between observers of $<1 \mathrm{ml}$. Twentythree non-identical lesions were caused by clicking in different parts of a heterogeneous lesion, which resulted in missing the SUVmax or SUVpeak of the lesion.

Interobserver Reliability ICC values for semi-automated MTVs were $0.43,0.86,0.96$, and 0.94 for the $41 \% \mathrm{MAX}$, $\mathrm{A} 50 \% \mathrm{P}, \mathrm{SUV} \geq 2.5$, and $\mathrm{SUV} \geq 4.0$ thresholds, respectively. Mean CoVs were $65.5 \%, 36.7 \%, 13.3 \%$, and $13.8 \%$, respectively (Table 1 ). When considering the $95 \%$ CIs of ICCs, only $\mathrm{SUV} \geq 2.5$ and $\mathrm{SUV} \geq 4.0$ showed excellent and good to excellent reliability, respectively.

For the MV2 and MV3 consensus methods, the mean CoVs were $22.7 \%$ and $33.5 \%$ and ICCs were 0.92 and 0.91 , respectively. Overall, fixed SUV threshold methods (SUV $\geq 2.5$ and $\mathrm{SUV} \geq 4.0$ ) showed least interobserver variability for MTV assessment in Workflow A. TLG showed similar ICCs and CoVs for these two methods.

Ease of Use Mean analysis time in Workflow A was 28.7 min per patient (range 5-63, Table 2). The most preferred method differed per patient and between observers (Table 3). A50\%P and SUV $\geq 4.0$ were most often chosen as "preferred segmentation" on a patient-level with success rates (rated as acceptable or good segmentations of visible tumor) ranging from 33 to $87 \%$ and $35-76 \%$, respectively. The mean success rate for the $41 \%$ MAX method ranged from 31 to $86 \%$ between observers. The success rates for the MV2 and MV3 methods, as scored by one observer, were $84 \%$ and $87 \%$, respectively. Although $\mathrm{SUV} \geq 2.5$ showed the highest observer reliability, this method was chosen only in 2 patients as the most preferred method by 1 observer. The mean success rate for this method ranged between 27 and $39 \%$ between observers. This method tended to overestimate the tumor volumes (Supplemental Figs. 1-2). Therefore, we decided to focus on the SUV $\geq 4.0$ method as preselection criterion.

\section{Workflow B; Preselection Strategy}

Lesion Selection The total number of selected tumor regions for observer 1,2 , and 3 was 76,76 , and 77 , respectively.

Seventy-two identical tumor regions were selected by all three observers.

Interobserver Reliability Workflow B is based on the $\mathrm{SUV} \geq 4.0$ threshold and showed good correlation with
SUV $\geq 4.0$ threshold of Workflow A with a Pearson correlation of 0.812 (after removing 4 volumes as outliers in 2 patients 0.995 , Fig. 1). Outliers were caused by one patient with many lesions, in whom the SUV $\geq 4.0$ threshold failed (large parts of the liver and spleen were included in this segmentation) and another with a large abdominal lesion that was interpreted as non-lymphoma by one observer. Complete agreement of the preselected volumes on a patient-level between all observers was found in six patients. The ICC value for generated MTVs in this workflow was excellent $(1.00,95 \% \mathrm{CI} 1.00-1.00)$ and the mean $\mathrm{CoV}$ was $2.3 \%$ (range $0-10.4 \%$, Table 1), with similar results for TLG.

Ease of Use Time to complete Workflow B ranged from 1 to 15 min (mean 7.3, Table 2). Preselected MTVs were rated as successful in seven, three, and four patients by the observers, respectively. They were classified as failures in zero, four, and six patients respectively.

\section{Workflow C; Manual Modification}

Effect of Manual Modification After manual modification of the preselected volumes the ICC of the final MTV was 0.92 (95\%CI 0.82-0.98, Table 1). Mean CoV for the final MTV was $16.7 \%$. Results for TLG again were similar, with excellent ICC values and good to excellent ICC values for MTV. The total time to perform this workflow ranged from 5 to 62 min (mean 22.2, Table 2).

Figure 2 shows the modified MTVs approved by a nuclear medicine physician (final MTV). Figure 3 shows a scatterplot of the correlation between the preselected and final MTV in Workflow C. Interestingly, the same outlier (patient 10) occurred as in Fig. 1, but contrary to this, two observers now decided to keep the entire liver in the preselection of Workflow $\mathrm{C}$ while they removed the liver uptake in Workflow B. For the final MTV, observer 2 had to remove the liver uptake after the check by the experienced NM physician. In another patient (patient 11), the preselection missed many small bone lesions, which were added manually. Figure 4 shows the Bland-Altman plot of the preselected and final MTV in Workflow C. The $95 \%$ limits of agreement ranged widely ( -525 to 458 ). The differences between preselected and final MTV did not have a normal distribution according to the SW test $(P=0.002)$. After excluding patients 10 and 11 (Figs. 3 and 4) described as outliers, the mean difference had a normal distribution $(P=$ $0.106)$. The plot shows both the original-as well as the recalculated $95 \%$ limits of agreement after exclusion of the outliers.

\section{Discussion}

We assessed the interobserver reliability and ease of use of three workflows for measuring MTV and TLG in 12 DLBCL patients and found that both improved when using 
Table 1. Interobserver reliability of semi-automated MTV and TLG assessment for the different workflows

\begin{tabular}{|c|c|c|c|c|c|c|}
\hline & \multicolumn{3}{|l|}{ MTV } & \multicolumn{3}{|l|}{ TLG } \\
\hline & Mean(range) & Mean CoV(range) & $\operatorname{ICC}(95 \% \mathrm{CI})$ & Mean(range) & Mean CoV(range) & $\mathrm{ICC}(95 \% \mathrm{CI})$ \\
\hline \multicolumn{7}{|c|}{ Workflow A (individual lesion selection) } \\
\hline $41 \% \mathrm{MAX}$ & $1106(33-4991)$ & $65.54(0-164.38)$ & $0.43(0.08-0.76)$ & $6236(471-21,431)$ & $54.57(0-151.84)$ & $0.37(0.02-0.72)$ \\
\hline $\mathrm{A} 50 \% \mathrm{P}$ & $550(34-4153)$ & $36.74(0-139.73)$ & $0.86(0.68-0.95)$ & $5736(245-45,441)$ & $26.76(0-118.26)$ & $0.93(0.82-0.98)$ \\
\hline $\mathrm{SUV} \geq 2.5$ & $2399(73-7404)$ & $13.34(0-54.21)$ & $0.96(0.91-0.99)$ & $15,902(347-55,588)$ & $7.11(0-33.81)$ & $0.99(0.98-1.00)$ \\
\hline $\mathrm{SUV} \geq 4.0$ & $1289(30-5688)$ & $13.78(0-83.59)$ & $0.94(0.86-0.98)$ & $13,617(220-50,068)$ & $11.32(0-82.52)$ & $0.97(0.93-0.99)$ \\
\hline MV2 & $1505(59-6258)$ & $22.68(0-83.59)$ & $0.92(0.80-0.97)$ & $14,422(301-51,908)$ & $15.84(0-82.52)$ & $0.97(0.91-0.99)$ \\
\hline MV3 & $927(33-4654)$ & $33.54(0-154.17)$ & $0.91(0.79-0.97)$ & $12,181(229-43,669)$ & $24.91(0-135.92)$ & $0.96(0.89-0.99)$ \\
\hline \multicolumn{7}{|c|}{ Workflow B (automated preselection) } \\
\hline $\mathrm{SUV} \geq 4.0$, Volume $\geq 3 \mathrm{ml}$ & $1004(23-5723)$ & $2.32(0-10.43)$ & $1.00(1.00-1.00)$ & $8446(189-50,779)$ & $1.85(0-7.49)$ & $1.00(1.00-1.00)$ \\
\hline \multicolumn{7}{|c|}{ Workflow C (automated preselection with manual modification) } \\
\hline Final MTV & $1115(53-5589)$ & 16.71(0-109.46) & $0.92(0.82-0.98)$ & $8610(284-48,079)$ & $13.33(0-111.83)$ & $0.97(0.93-0.99)$ \\
\hline
\end{tabular}

$M V$, majority vote; $M T V$, metabolic tumor volume; $\mathrm{CoV}$, coefficient of variation; $I C C$, intraclass correlation coefficient; $C I$, confidence interval; $T L G$, total lesion glycolysis

a fully automated preselection approach to measure MTV and TLG (using SUV $\geq 4.0$ and volume $\geq 3 \mathrm{ml}$ ).

Ilyas et al. [10] compared three MTV segmentation methods (SUV $\geq 2.5,41 \% \mathrm{MAX}$ and PERCIST) in patients with DLBCL and concluded that data-driven optimal cutoff values for separation of patients into a good and a poor prognosis group were largely dependent on the method used, but these datadriven cutoff values had comparable prognostic accuracy. In a subset of 50 patients evaluated by two observers, they found that interobserver reliability was excellent (ICC $>0.98$ ). They further reported a mean analysis time ranging between 2.7 and 6.2 min for the 3 methods [10]. The data-analysis in our study took more time, possibly due to less experience of the observers with the software and the datasheets that had to be completed, which was not included in the time per patient reported in Ilyas study. Yet, also in our study, we found that when total metabolic tumor volume was derived using the preselection and when unwanted normal tissue uptake could be removed and missed lesions could be added by single mouse clicks, the overall processing time was typically less than $5 \mathrm{~min}$. In cases where manual corrections or manual definitions of the VOIs were needed, processing time could well exceed $20 \mathrm{~min}$.

Another important finding in the Ilyas study and our study is that the $\mathrm{SUV} \geq 2.5$ method showed the highest interobserver reliability. Interestingly though, the observers in our study considered that $\mathrm{SUV} \geq 2.5$ often overestimated the volume compared with other methods and was almost never chosen as their preferred method on a patient-level.
However, a recent study (partly by the same authors) showed that a slightly higher threshold (SUV $\geq 4.0$ ) outperformed the SUV $\geq 2.5$ in terms of success rate [23].

A recent phantom and patient study in primary mediastinal B cell lymphoma that compared four different MTV methods found that $S U V \geq 2.5$ resulted in an overestimation, particularly at high SUV values and 41\%MAX underestimated MTV when there were high levels of heterogeneity [24].

In a publication by Meignan et al. [12], two observers used two percentage-based methods for MTV assessment in DLBCL (41\%MAX and a variable SUVmax threshold that visually resulted in optimal segmentations). They found substantial reliability of 0.99 for the $41 \% \mathrm{MAX}$ threshold and poor reliability of 0.86 for the variable percentage of SUVmax according to Lin's concordance correlation coefficient. This study also suggests that reliability decreased with an increasing level of user interaction.

Based on the ratings of individual lesions it could be argued that no single semi-automated segmentation method performed well for every patient and within every lesion of that patient. Lymphoma sites can be difficult to segment because of heterogeneity within and between lesions. Some patients have many lesions, making it almost impossible to delineate each lesion. Besides that, it should be noted that a visual check of the generated segmentation by an experienced nuclear medicine physician or radiologist is necessary if a semi-automated method is applied, as was illustrated by the outliers in this pilot study. For example, patient 10 showed a large difference between the three workflows

Table 2. Mean analysis time for the different workflows in minutes (mean \pm standard deviation (range))

\begin{tabular}{llll}
\hline Workflow & A individual lesion selection $(n=12)$ & B automated preselection $(n=12)$ & C with manual modification $(n=12)$ \\
\hline Observer 1 & $29.1 \pm 20.8(5-63)$ & $7.2 \pm 3.7(3-15)$ & $23.3 \pm 13.4(5-45)$ \\
Observer 2 & Not reported* & Not reported* & $26.7 \pm 15.6(10-62)$ \\
Observer 3 & $28.2 \pm 13.7(15-60)$ & $7.3 \pm 3.5(1-12)$ & $16.7 \pm 9.7(8-42)$ \\
Mean & $28.7^{\dagger}$ & $7.3^{\dagger}$ & 22.2 \\
\hline
\end{tabular}

*Observer 2 summed the total time for Workflow A + B; mean $27.3 \pm 19.2$ (7-75) minutes

${ }^{\dagger}$ Mean value based on 2 observers 
Table 3. Most preferred method per observer for Workflow A

\begin{tabular}{llll}
\hline Patient & Observer 1 & Observer 2 & Observer 3 \\
\hline 1 & $41 \% \mathrm{MAX}$ & $41 \% \mathrm{MAX}$ & $\mathrm{SUV} \geq 4.0$ \\
2 & $41 \% \mathrm{MAX}$ & $41 \% \mathrm{MAX} / \mathrm{A} 50 \% \mathrm{P} / \mathrm{SUV} \geq 4.0$ & $\mathrm{SUV} \geq 2.5$ \\
3 & A50\%P & $41 \% \mathrm{MAX}$ & $\mathrm{SUV} \geq 4.0$ \\
4 & $\mathrm{SUV} \geq 4.0$ & A50\%P & $\mathrm{SUV} \geq 4.0$ \\
5 & $\mathrm{SUV} \geq 4.0$ & A50\%P & $\mathrm{SUV} \geq 4.0$ \\
6 & $\mathrm{SUV} \geq 4.0$ & $41 \% \mathrm{MAX} / \mathrm{A} 50 \% \mathrm{P}$ & $\mathrm{SUV} \geq 4.0$ \\
7 & $\mathrm{~A} 50 \% \mathrm{P}$ & A50\%P & $\mathrm{SUV} \geq 2.5$ \\
8 & $\mathrm{~A} 50 \% \mathrm{P}$ & $41 \% \mathrm{MAX} / \mathrm{A} 50 \% \mathrm{P}$ & $\mathrm{SUV} \geq 4.0$ \\
9 & $\mathrm{~A} 50 \% \mathrm{P}$ & $41 \% \mathrm{MAX}$ & $\mathrm{SUV} \geq 4.0$ \\
10 & $\mathrm{~A} 50 \% \mathrm{P}$ & A50\%P & $\mathrm{A} 50 \% \mathrm{P}$ \\
11 & $\mathrm{SUV} \geq 4.0$ & $41 \% \mathrm{MAX}$ & $\mathrm{A} 50 \% \mathrm{P}$ \\
12 & $41 \% \mathrm{MAX} / \mathrm{SUV} \geq 4.0$ & $41 \% \mathrm{MAX}$ & $\mathrm{A} 50 \% \mathrm{P}$ \\
\hline
\end{tabular}

Each observer indicated their "preferred segmentation" for individual lesions. The most preferred method per patient was defined as the method most often noted as "preferred segmentation"

(Figs. 1 to 3). It appeared that the decision whether the liver was involved or not was the main reason for the large differences in the assessments. Both the observers and the NM physicians did not agree on the question of whether the liver was involved or not. In clinical practice, access to additional clinical information (e.g., physical examination or lab results) may help to support the decision whether a site is involved or not. This situation illustrates the importance of the development of clear clinical criteria, definitions, and guidelines for lesion selection in PET/CT studies of patients with different lymphoma types [25].
We also compared the results of the observers (who were clinicians, but not NM physicians) before and after the check of the NM physician. It appeared that only small lesions were added, and in a few patients, physiological uptake was erroneously included in MTV, again supporting the need for checking of results by a NM physician.

This study has strengths and limitations that should be taken into account when interpreting the results. First, we deliberately selected patients with a large variation in number and size of lesions. This might be a strength because it represents examples of different challenges that can occur when analyzing MTV in lymphoma, but this could give a higher prevalence of difficult cases compared with the general DLBCL cohort. However, according to the three experienced nuclear medicine physicians, the dataset was representative of a general DLBCL cohort, even though we selected a relatively small number of patients.

Another strength is the comparison of different workflows for MTV and TLG assessment and their impact on interobserver reliability. Most studies acknowledge the difficulties in the assessment of multiple lymphoma lesions. Some used boxes or VOIs to constrain individual tumors [6, $8,12]$, or limited segmentation to a representative maximum of 5 lymphoma lesions [26], but none of these studies compared such strategies with another workflow.

A limitation is the dependency of the ICC values on the range of MTV values in the population [21]. This is present in other MTV studies as well and hampers comparability of

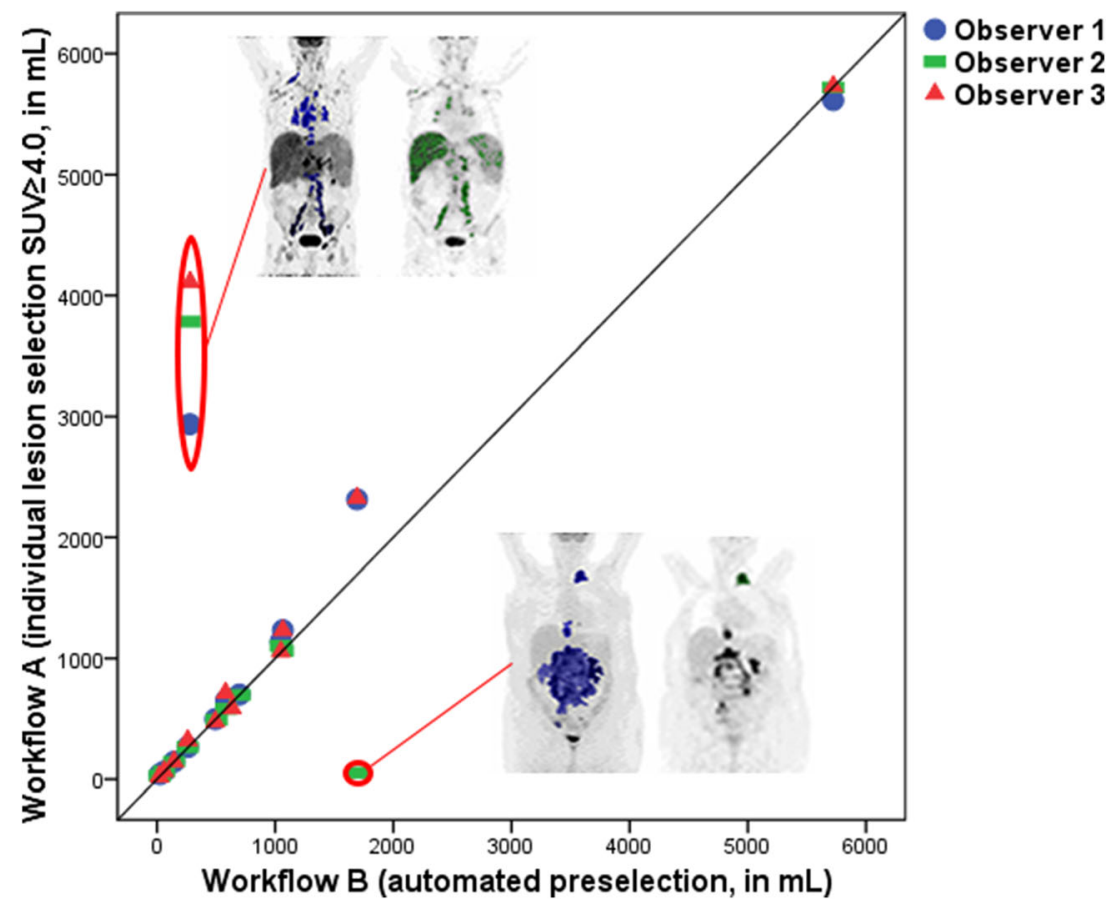

Fig. 1. Scatterplot of MTV for Workflow A (user-defined selection with $S U V \geq 4.0$ ) and Workflow $B$ (automated preselection). PET images represent examples of different MTV interpretations between the workflows. Top left images (patient 10): Workflow B contains only lymphoma lesions around the large vessels (left), while in Workflow A, the liver and spleen were also included in the lesion selection (right). Bottom right images (patient 8): in Workflow B, the large lesion was selected (left), while it was interpreted as not being lymphoma in Workflow A (right). 


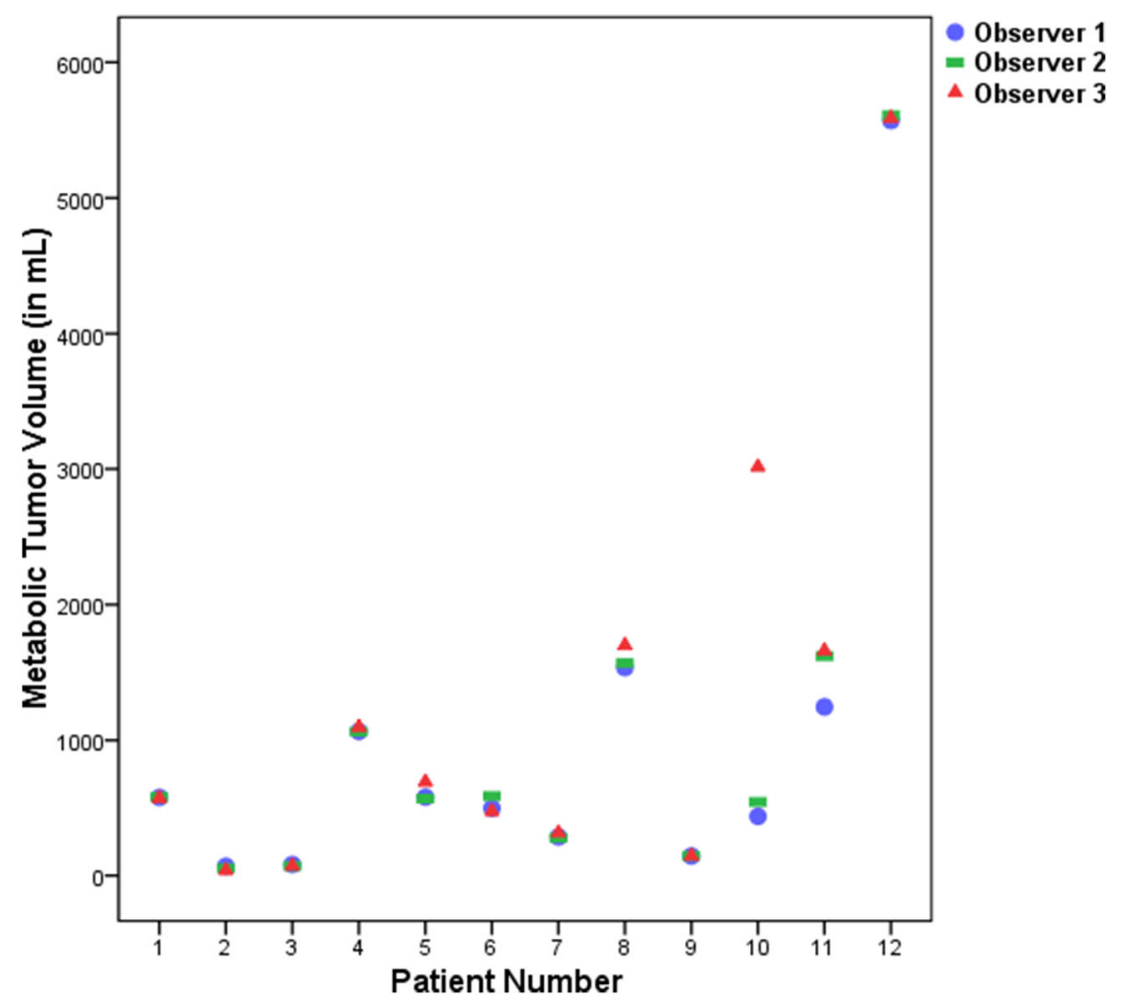

Fig. 2. Scatterplot of final MTV assessment in Workflow C.

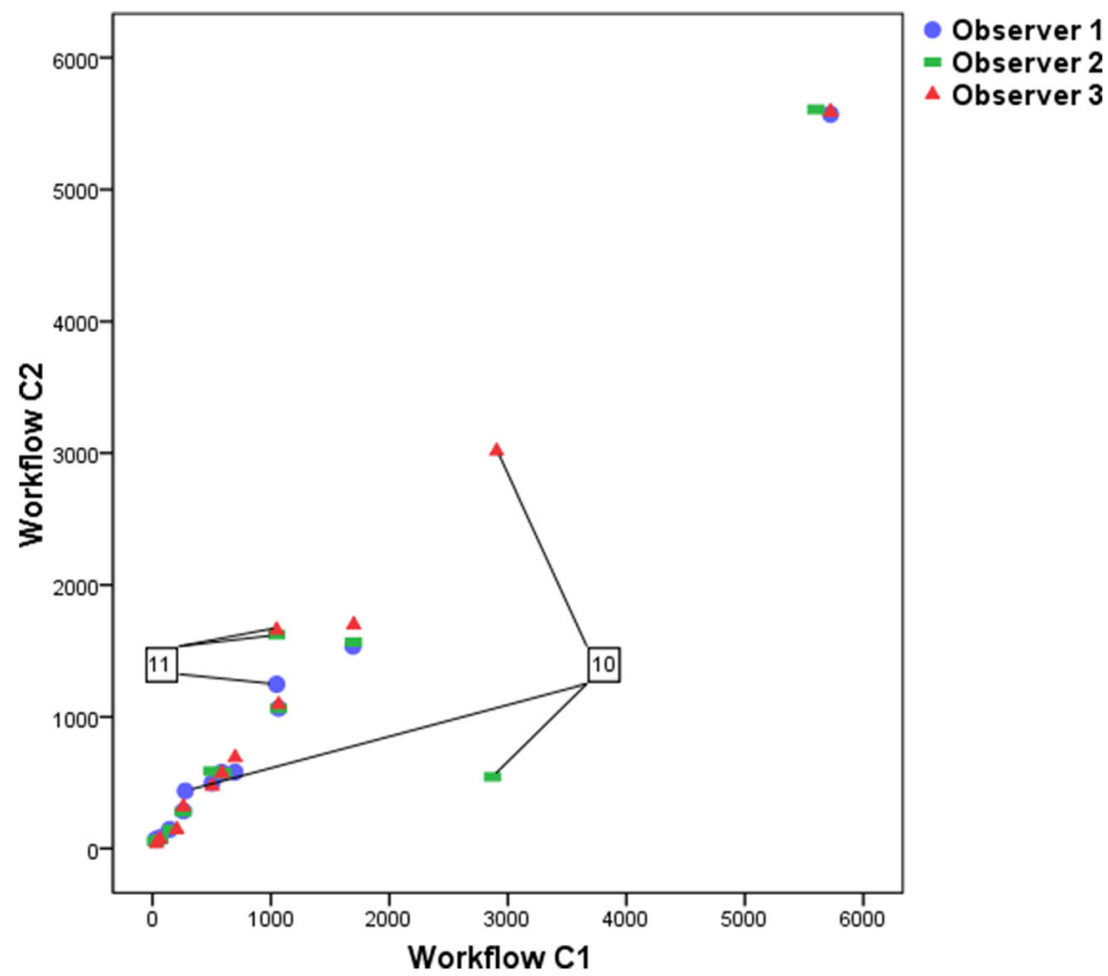

Fig. 3. Scatterplot of MTV assessment in Workflow C (automated preselection before (C1) - and final MTV after manual modification (C2), in milliliters). Datapoints from two challenging patients (patients 10 and 11) are indicated by lines. The numbers in the boxes refer to the patient numbers described in the main text. 


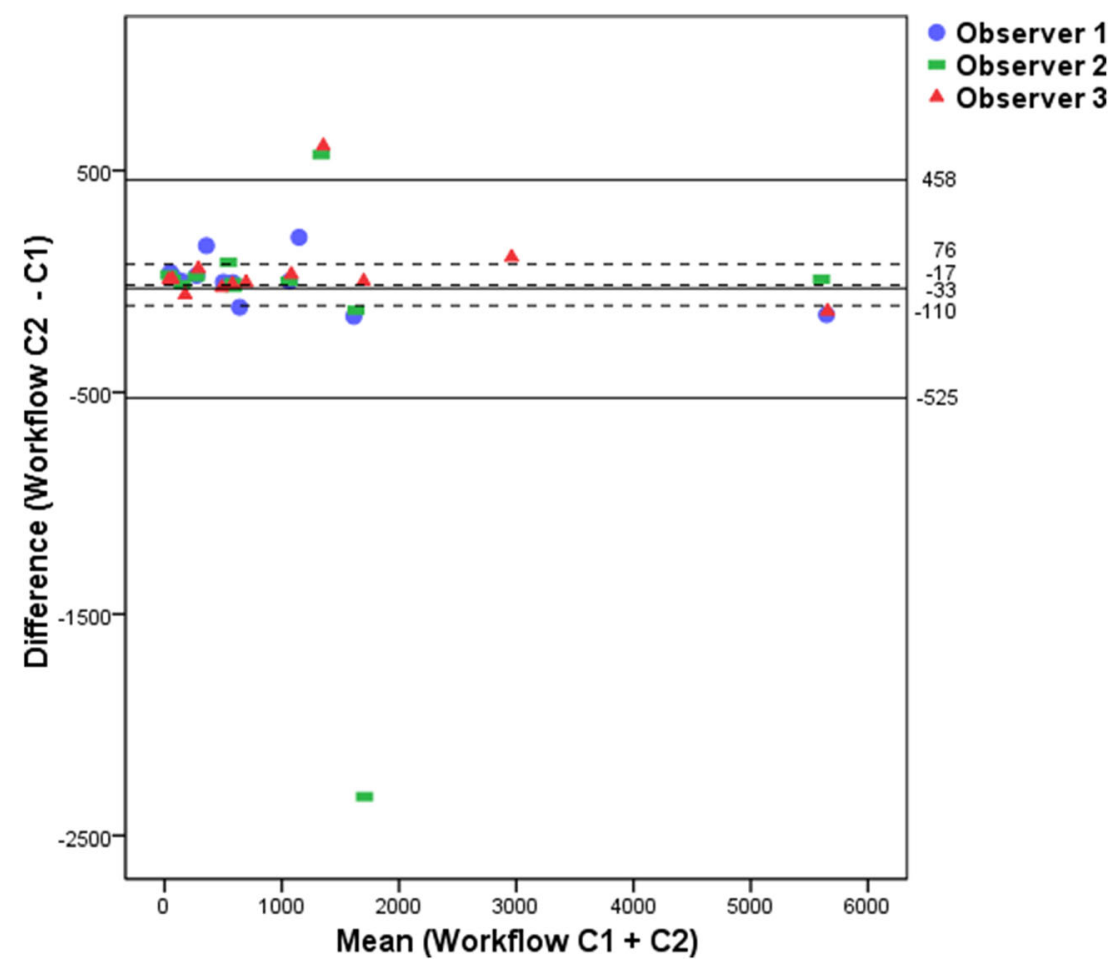

Fig. 4. Bland-Altman plot showing effect of manual modification of MTV assessment in Workflow C (automated preselection before (C1) —and final MTV after manual modification (C2)). Solid line: mean value, upper- and lower limit of agreement without exclusion of outliers. Dashed line: mean value, upper- and lower limit of agreement after exclusion of patients 10 and 11.

ICCs within and between studies. Therefore, we also presented CoVs and Bland-Altman plots which are not dependent on the variability of MTV values among patients.

Finally, a preselection strategy as suggested in this study is not yet widely available in other commercially available (clinical) software tools but could be implemented relatively easily after validation in a larger patient cohort.

Future research should focus on the comparison of a preselection strategy in a larger patient cohort with different segmentation methods, their success rates, and the effect on the prognostic value of MTV and TLG measurements. A possible solution for the problem that none of the methods will be satisfactory in each patient and for each lesion could be the use of a MV approach, which should be investigated further. In addition, the effect of reconstruction settings, different uptake times, and effect of adding small lesions on the accuracy of MTV and TLG measurements should be addressed.

\section{Conclusions}

A semi-automated workflow based on individual lesion selection (Workflow A) is not recommended, because of the large differences observed in lesion selection. Using a fully automated preselection ( $\mathrm{SUV} \geq 4.0$ and volume $\geq 3 \mathrm{ml}$, Workflow B) of lesions improved interobserver reliability and ease of use of MTV and TLG assessment in DLBCL patients. Subsequent manual modification (Workflow C) is necessary for some patients, but this reduced interobserver reliability which may need to be balanced against any potential improvement of prognostic accuracy.

Acknowledgments. The authors thank all the patients who took part in the trials and the collaborating investigators that kindly supplied their trial data.

Funding Information. The PETRA study is supported by the Alpe d'HuZes/ KWF fund, provided by the Dutch Cancer Society (\#VU2012-5848). Alpe d'HuZes/KWF did not have any role in the study design, data collection, analysis, interpretation, and writing of the manuscript.

\section{Compliance with Ethical Standards}

\section{Conflict of Interest}

CNB, FR, IK, SP, YWSJ, GJCZ, SM, OSH, JMZ, and HCWdV declare that they have no conflict of interest. SFB has received research funding from Hermes Medical Solutions. SFB acknowledges support from the National Institute for Health Research and Social Care (NIHR) [RP-2-16-07-001]. King's College London and UCL Comprehensive Cancer Imaging Centre is funded by the CRUK and EPSRC in association with the MRC and Department of Health (England).The views expressed are those of the author(s) and not necessarily those of the NHS, the NIHR or the Department of Health and Social Care. RB is a member of the EARL steering board (without financial benefits).

\section{Ethical Approval}

All procedures performed in studies involving human participants were in accordance with the ethical standards of the institutional and/or national research committee and with the 1964 Helsinki declaration and its later amendments or comparable ethical standards.

\section{Informed Consent}

Informed consent was obtained from all individual participants included in the study. 
Open Access This article is licensed under a Creative Commons Attribution 4.0 International License, which permits use, sharing, adaptation, distribution and reproduction in any medium or format, as long as you give appropriate credit to the original author(s) and the source, provide a link to the Creative Commons licence, and indicate if changes were made. The images or other third party material in this article are included in the article's Creative Commons licence, unless indicated otherwise in a credit line to the material. If material is not included in the article's Creative Commons licence and your intended use is not permitted by statutory regulation or exceeds the permitted use, you will need to obtain permission directly from the copyright holder. To view a copy of this licence, visit http:// creativecommons.org/licenses/by/4.0/.

\section{References}

1. Pfreundschuh M, Ho AD, Cavallin-Stahl E et al (2008) Prognostic significance of maximum tumour (bulk) diameter in young patients with good-prognosis diffuse large-B-cell lymphoma treated with CHOP-like chemotherapy with or without rituximab: an exploratory analysis of the MabThera International Trial Group (MInT) study. Lancet Oncol 9:435-444

2. Barrington SF, Mikhaeel NG, Kostakoglu L et al (2014) Role of imaging in the staging and response assessment of lymphoma: consensus of the International Conference on Malignant Lymphomas Imaging Working Group. J Clin Oncol 32:3048-3058

3. Cheebsumon P, Boellaard R, de Ruysscher D et al (2012) Assessment of tumour size in PET/CT lung cancer studies: PET- and CT-based methods compared to pathology. EJNMMI Res 2:56

4. Frings V, de Langen AJ, Smit EF et al (2010) Repeatability of metabolically active volume measurements with $18 \mathrm{~F}-\mathrm{FDG}$ and $18 \mathrm{~F}-$ FLT PET in non-small cell lung cancer. J Nucl Med 51:1870-1877

5. Wang XY, Zhao YF, Liu Y, Yang YK, Zhu Z, Wu N (2017) Comparison of different automated lesion delineation methods for metabolic tumor volume of $18 \mathrm{~F}-\mathrm{FDG}$ PET/CT in patients with stage I lung adenocarcinoma. Medicine (Baltimore) 96(51):e9365

6. Cottereau AS, Lanic H, Mareschal S et al (2016) Molecular profile and FDG-PET/CT total metabolic tumor volume improve risk classification at diagnosis for patients with diffuse large B-cell lymphoma. Clin Cancer Res 22:3801-3809

7. Mikhaeel NG, Smith D, Dunn JT et al (2016) Combination of baseline metabolic tumour volume and early response on PET/CT improves progression-free survival prediction in DLBCL. Eur J Nucl Med Mol Imaging 43:1209-1219

8. Sasanelli M, Meignan M, Haioun C et al (2014) Pretherapy metabolic tumour volume is an independent predictor of outcome in patients with diffuse large B-cell lymphoma. Eur J Nucl Med Mol Imaging 41:2017-2022

9. Song MK, Chung JS, Shin HJ et al (2012) Clinical significance of metabolic tumor volume by PET/CT in stages II and III of diffuse large B cell lymphoma without extranodal site involvement. Ann Hematol 91:697-703

10. Ilyas H, Mikhaeel NG, Dunn JT et al (2018) Defining the optimal method for measuring baseline metabolic tumour volume in diffuse large B cell lymphoma. Eur J Nucl Med Mol Imaging 45:1142-1154

11. Kurtz DM, Green MR, Bratman SV et al (2015) Noninvasive monitoring of diffuse large B-cell lymphoma by immunoglobulin high-throughput sequencing. Blood 125:3679-3687
12. Meignan M, Sasanelli M, Casasnovas RO et al (2014) Metabolic tumour volumes measured at staging in lymphoma: methodological evaluation on phantom experiments and patients. Eur J Nucl Med Mol Imaging 41:1113-1122

13. Kostakoglu L, Chauvie S (2018) Metabolic tumor volume metrics in lymphoma. Semin Nucl Med 48:50-66

14. Cottereau AS, Hapdey S, Chartier L et al (2017) Baseline total metabolic tumor volume measured with fixed or different adaptive thresholding methods equally predicts outcome in peripheral $\mathrm{T}$ cell lymphoma. J Nucl Med 58:276-281

15. Kanoun S, Tal I, Berriolo-Riedinger A et al (2015) Influence of software tool and methodological aspects of total metabolic tumor volume calculation on baseline [18F]FDG PET to predict survival in Hodgkin lymphoma. PLoS One 10:e0140830

16. Boellaard R (2018) Quantitative oncology molecular analysis suite: ACCURATE [abstract]. J Nucl Med 59(suppl.1):1753

17. Frings V, van Velden FH, Velasquez LM et al (2014) Repeatability of metabolically active tumor volume measurements with FDG PET/CT in advanced gastrointestinal malignancies: a multicenter study. Radiology. 273:539-548

18. Schaefer A, Vermandel M, Baillet $C$ et al (2016) Impact of consensus contours from multiple PET segmentation methods on the accuracy of functional volume delineation. Eur J Nucl Med Mol Imaging 43:911924

19. McGraw KO, Wong SP (1996) Forming inferences about some intraclass correlation coefficients. Psychol Methods 1:30-46

20. Koo TK, Li MY (2016) A guideline of selecting and reporting intraclass correlation coefficients for reliability research. J Chiropr Med 15:155-163

21. Portney LG, Watkins MP (2009) Intraclass correlation coefficient (ICC). In: Foundations of clinical research: applications to practice. Pearson Prentice Hall, Upper Saddle River, New Jersey, pp 588-598

22. Bland JM, Altman DG (1986) Statistical methods for assessing agreement between two methods of clinical measurement. Lancet. $1: 307-310$

23. Barrington SF, de Vet HCW, Mikhaeel NG, et al. (2018) Automated segmentation of total tumour burden in DLBCL: which method is most successful? $7^{\text {th }}$ PILM, Menton [Abstract V1] https:// $\mathrm{dr}$ i v e.g o o g 1 e . c o m/d r i v e / f o $1 \mathrm{de} \mathrm{r} \mathrm{s/}$ 1a0q5uYLBx6HqcUjBxTryiWPUOWFTJGLG;

24. Ceriani L, Milan L, Johnson PWM et al (2019) Baseline PET features to predict prognosis in primary mediastinal $\mathrm{B}$ cell lymphoma: a comparative analysis of different methods for measuring baseline metabolic tumour volume. Eur J Nucl Med Mol Imaging 46:13341344

25. Barrington SF, Meignan MA (2019) Time to prepare for risk adaptation in lymphoma by standardising measurement of metabolic tumour burden. J Nucl Med 60:1096-1102

26. Parvez A, Tau N, Hussey D, Maganti M, Metser U (2018) (18)F-FDG $\mathrm{PET} / \mathrm{CT}$ metabolic tumor parameters and radiomics features in aggressive non-Hodgkin's lymphoma as predictors of treatment outcome and survival. Ann Nucl Med 32:410-416

Publisher's Note Springer Nature remains neutral with regard to jurisdictional claims in published maps and institutional affiliations. 